\title{
Correlational cuing as a function of target complexity and target-flanker similarity
}

\author{
ASHER COHEN, AHUVA FUCHS, ATARA BAR-SELA, YARON BRUMBERG, and HAGIT MAGEN \\ The Hebrew University, Jerusalem, Israel
}

\begin{abstract}
It is generally assumed that the correlational cuing effect (CE) between targets and correlated flankers is due to learning association between the flankers and their correlated responses. The present study challenges this view. Experiment 1 shows that the $\mathrm{CE}$ for targets composed of color is eliminated as soon as the correlation is removed. Experiment 2 shows that the $\mathrm{CE}$ during training is not due to association of the flankers with responses. Experiment 3 shows that at least some of the $\mathrm{CE}$ during training with the correlation is due to repetition priming of the display. Experiment 4 replicates the results of Experiment 1 for orientation targets. In Experiments 5-7, more typical tasks with letter targets are examined, and it is demonstrated that preexperimental similarity between targets and correlated flankers is crut cial. The $\mathrm{CE}$ for correlated but dissimilar target-flanker pairs, similar to that for color and orientation targets, is confined to on-line processes that occur during training. The CE is transferred, however, for correlated and similar target-flanker pairs. We propose that, at least for the simple stimulus to response mapping used in our study, the CE is not due to learning at all. Instead it is due to (1) on-line processes, such as repetition priming, that occur during training with the correlation and (2) a regular flanker effect (see, e.g., B. A. Eriksen \& C. W. Eriksen, 1974) that occurs for similar target-flanker pairs.
\end{abstract}

Many visual tasks involve situations in which a target appears among distractors that are not directly relevant to the task. A major issue in visual cognition is the extent to which processing of a target in a known location is affected by the identity of distractors located elsewhere in the visual field.

A popular paradigm for this purpose is the flanker paradigm. In a typical flanker task (see, e.g., C. W. Eriksen \& B. A. Eriksen, 1979), the subjects are required to respond to a target appearing in a known location, often at the center of the screen. Two stimuli (e.g., the letters F and S) are assigned to one response, and two other stimuli (e.g., $X$ and C) are assigned to a second response. The target is flanked by two stimuli on its sides. Although the identity of the flankers is irrelevant to the task, the subjects are typically unable to ignore it (C. W. Eriksen \& B. A. Eriksen, 1979; Miller, 1991; Yantis \& Johnston, 1990). They respond faster in the congruent condition, in which the flankers belong to the same response set as the target (e.g., F flanked by Ss) than in the incongruent condition, in which the flankers belong to the other response set (e.g., F flanked by Xs). This response latency difference between the congruent and incongruent conditions is known as the congruency effect.

A number of authors have suggested that the presence of the flanker congruency effect has important implica-

This study was supported by a grant from Israel Foundations Trustees (1996-1998) to A.C. and by US PHS Grant MH5I400 to Robert Rafal, Richard Ivry, and A.C. We thank John Flowers, Art Kramer, J. Toby Mordkoff, Rachel Shoup, and Naomi Goldblum for helpful comments on earlier versions of the manuscript. Correspondence concerning this article should be addressed to A. Cohen. Department of Psychology, the Hebrew University, Jerusalem 91905, Israel (e-mail: msasher@mscc. huji.ac.il). tions for determining the locus of attentional processes (e.g., Kahneman \& Treisman, 1984; Miller, 1991; Yantis \& Johnston, 1990). In particular, it has been suggested that the flanker congruency effect is not mediated by attentional processes and is, thus, a good indication of preattentive processes (see, e.g., Van der Heijden, 1981). One problematic aspect of the flanker task for this issue is that the flankers in the congruent and incongruent conditions are taken from the same set of stimuli as are the targets. Because the subjects expect these stimuli to appear, the appearance of the flankers may attract attention to their locations. Thus, it is possible that attentional processes cause the flanker congruency effect.

Miller (1987) designed a somewhat different paradigm, the correlational cuing paradigm, to overcome this problem. As in the flanker paradigm, the subjects were required to respond to a central target and ignore irrelevant flankers. Unlike the situation in the flanker task, however, the targets and flankers belonged to different sets of stimuli. The main manipulation in Miller's (1987) task was the degree of the correlation between the targets and the flankers. For example, the subjects were required to discriminate between two targets (e.g., $\mathrm{X}$ and $\mathrm{O}$ ), while two other letters (e.g., F and E) served as flankers. One of the targets (e.g., X) was presented most often with one of the flankers (e.g., F) and only occasionally with the other flanker (E). The situation was reversed for the other target (i.e., it was presented most often with the flanker $E$ and only occasionally with the flanker $F$ ).

An appealing aspect of this correlational cuing paradigm is that the flankers are not directly relevant for the task and the subjects can completely ignore them. However, Miller (1987) found that subjects did not ignore the 
flankers and, in fact, took advantage of the correlations. They responded faster in the high-correlation condition, in which the target was presented with its more frequent flanker, than in the low-correlation condition, in which the target was presented with its less frequent flanker. Miller (1987) showed that this correlational cuing effect (CE) occurred even when subjects were not at all aware of the flankers' identity (but see Schmidt \& Dark, 1998).

What is the locus of this CE? One potential explanation is simply the relative frequency of the different displays. In the example above, there are many more trials in which the target appeared with its high-correlation flankers than trials in which it appeared with its low-correlation display. Moreover, the likelihood of two consecutive trials with identical displays is much higher for high-correlation displays than for low-correlation displays. Much evidence suggests that repetition of a trial leads to a faster response (see, e.g., Bertelson, 1963; Kornblum, 1969). Miller (1987) showed, however, that subjects are faster in high-correlation conditions, even when the display frequency is controlled.

It appears, therefore, that subjects are able to learn the correlation between targets and irrelevant distractors. What is the nature of the learned correlation? One possibility is that subjects learn the correlation between the flankers and the targets. Alternatively, subjects may have learned the correlation between the flankers and the required response. In the above examples, these two possibilities were confounded. In several ingenious experiments, Miller (1987) showed that the learned correlation is between the flankers and the response assignment. For example, in one of his experiments, Miller (1987) initially trained subjects on a typical correlational cuing task. In subsequent blocks, he changed the task and required the subjects to respond to the stimuli that had served as flankers during the training blocks. Some of these stimuli were assigned a response that had been positively correlated with them during the training blocks, whereas other stimuli were assigned a response that had been negatively correlated with them during the training blocks. Miller (1987) found that reaction time (RT) was faster in the former than in the latter, suggesting that, during the training blocks, the subjects had learned to associate the flankers with their correlated responses.

These findings have profound implications for visual tasks involving targets and distractors. Although the degree to which subjects can ignore the flankers in the correlational cuing paradigm has subsequently been débated (e.g., Carlson \& Flowers, 1996; Lavie, 1995; Paquet \& Craig, 1997; Paquet \& Lortie, 1990; Schmidt \& Dark, 1998), it has generally been assumed that at least some processing of irrelevant flankers is mandatory.

Two additional assumptions go beyond the simple (if important) assumption of mandatory flanker processing. First, it is assumed that the presence of a CE is an indication that subjects learned the correlations between flankers and responses and formed associations accordingly. A second assumption is that the congruency effect in the typical flanker task mentioned earlier (see, e.g., C. W. Eriksen \&
B. A. Eriksen, 1979) is caused by the same factors as is the CE. There is strong evidence that a large part of the flanker congruency effect is due to response selection processes (see Cohen \& Shoup, 1993, 1997, for reviews), and, as mentioned earlier, there is also evidence that the CE is response based (Miller, 1987). Consequently, the findings from the two paradigms have often been treated as interchangeable (e.g., Schmidt \& Dark, 1998).

The main purpose of the present paper is to challenge the latter two assumptions concerning the correlational cuing paradigm. In the first part of the paper (Experiments $1-4)$, we present evidence that CEs with targets defined by simple properties, such as color and orientation, are not due to learning long-lasting associations between the target and the flankers. Instead, the effects appear to be caused primarily by on-line local processes (i.e., processes that do not result in learning) that occur during training with the correlation (e.g., display frequency). We provide evidence (Experiment 3) that one major source of the effect is repetition priming of the display. In the second part of the paper (Experiments 5-7), we examine correlational cuing with targets defined by letters. We suggest that the effect obtained with letters is primarily due to two factors: on-line local processes that occur during training and similarity between target and distractors. In particular, we show that when the similarity between targets and high-correlation distractors is low, the entire CE is due to local processes. When the similarity between targets and high-correlation flankers is relatively high, a second effect is present as well. We speculate that this second effect is not the learning of associations, as is commonly believed, but is similar to the congruency effect obtained in typical flanker studies, and Miller (1987) has presented evidence that the $\mathrm{CE}$ is not due to local contingencies but to association between the high-correlation flankers and the required response; we suggest that the presence of flanker congruency owing to similarity in Miller's (1987) experiments may have led to the results obtained in his study.

\section{EXPERIMENT 1}

The purpose of this experiment was to examine correlational cuing with two targets defined by their color. The targets were flanked horizontally by stimuli from a different set of two colors. The two flankers in each display were always identical. One target appeared most frequently ( $90 \%$ of its trials) with one of the flankers and less frequently ( $10 \%$ of its trials) with the other flanker. The reverse was true for the other target. To control for pair-wise differences in perceptual similarity between the two sets of colors, we counterbalanced the pairing of the targets and distractors (see Table 1). We refer to the two resulting pairings as Versions 1 and 2.

All the subjects in this experiment received a practice block followed by four training blocks with this correlation manipulation. Following the training blocks, the subjects received a single transfer block in which there was no 
Table 1

The Target-Flanker Pairs Used in Experiment $1 \mathrm{~A}$ and $1 \mathrm{~B}$

\begin{tabular}{ccc}
\hline Version & High-Correlation Pairs & Low-Correlation Pairs \\
\hline 1 & blue-red & blue-green \\
& yellow-green & yellow-red \\
2 & blue-green & blue-red \\
& yellow-red & yellow-green \\
\hline
\end{tabular}

Note-The first color is that of the target, whereas the second is that of the flanker.

correlation between the targets and the flankers. That is, in this block each target appeared equally often with the two flankers. The purpose of the transfer block was to examine the nature of residual learning from the training blocks.

To control for possible general practice effects, we also used a control task. The same control task was used in Versions 1 and 2. In this control task, the subjects received the same amount of training, but the training blocks were the same as the transfer block. That is, each target appeared equally often with the two flankers throughout the experiment. Note that the targets were identical for all the tasks. The instructions for all the subjects were identical as well. The subjects were simply told that there would be a central target and two irrelevant flankers and were told the mapping of the targets to the responses.

\section{Method}

Subjects. Sixty subjects from the Hebrew University were tested in one session, either as part of their course requirements or for a payment of approximately $\$ 5$ per session. Thirty subjects participated in Version 1, and the remaining subjects in Version 2. Fifteen subjects in each group were in the experimental condition, whereas the others were in the control condition.

Apparatus. The stimuli were presented on a NEC MultiSynch $4 \mathrm{E}$ color monitor controlled by an Intel 486 microcomputer. The subjects were tested in a dimly lit room and viewed the display from a distance of $100 \mathrm{~cm}$ with the aid of a chin rest. The subjects responded by using their dominant hand to press one of two microswitch keys mounted on a response board interfaced with the computer.

Stimuli and Design. Each display in this experiment consisted of a central target and two flankers. All the stimuli used in the experiment were colored vertical lines subtending approximately $0.52^{\circ}$ of visual angle in length, with a luminance of approximately $30 \mathrm{~cd} / \mathrm{m}^{2}$ against a dark background $\left(0 \mathrm{~cd} / \mathrm{m}^{2}\right)$. The two targets were blue (Response 1) and yellow (Response 2) vertical lines. The flankers were red and green vertical lines. The center-to-center distance between the target and each of the two flankers was approximately $0.86^{\circ}$ of visual angle.

The difference between the various conditions was in the proportion of trials in which the two targets were paired with the two flankers. In the experimental conditions of Versions 1 and 2, the four possible target -flanker pairs were divided into two high-correlation and two low-correlation pairs. The high-correlation pairs were presented on $90 \%$ of the trials, and the low-correlation pairs on $10 \%$ of the trials. The two pairs within each correlation group were presented equally often. Each training block (as well as the practice block) consisted of 160 trials. Within each block, the two high-correlation pairs appeared in 72 trials each, and the two low-correlation pairs in 8 trials each. The transfer block for all the groups and the training blocks for the control conditions consisted of 160 trials as well. However, within these blocks. each of the four pairs was presented in 40 trials. The order of the trials within each block was determined randomly. Each subject first received a practice block, followed by four training blocks and a transfer block of 160 trials each.
Note that the control conditions of Versions 1 and 2 are identical. We ran two groups of subjects to make sure of independent comparisons for the two experimental conditions.

Procedure. At the beginning of each trial, an achromatic asterisk, serving as a fixation point, was presented in the center of the visual screen. After $500 \mathrm{msec}$, the asterisk was replaced by the stimulus display. The display remained visible until the subject pressed one of the two response keys. The subjects were instructed to respond as fast as they could, while minimizing mistakes. The screen turned blank immediately following the subject's correct response until the appearance of the asterisk for the next trial. The message ERROR was presented on the screen for $500 \mathrm{msec}$ following incorrect responses. In either case, the intertrial interval was $1,000 \mathrm{msec}$.

\section{Results and Discussion}

In all the experiments reported in this paper, we took several steps before analyzing the RTs. First, we did not analyze incorrect responses. Second, we eliminated RTs more than three standard deviations above the mean. Finally, the pattern of the proportion of errors in all the experiments was generally low and paralleled that of the RTs. This indicates that the pattern of the RT results cannot be attributed to a speed-accuracy tradeoff. Thus, although we report the proportions of errors in the various conditions, we did not analyze them statistically.

Table 2 presents the mean RTs and proportion of errors in the training blocks (collapsed over the four blocks) and the transfer block for the two experimental and the two control groups. Note that the control group did not really have different correlation conditions. The high-correlation and low-correlation conditions for each of the control groups were based on the experimental condition to which the control group was matched. For example, the highcorrelation condition for the control group in Version 1 consisted of the two target-flanker pairs that were highly correlated in the experimental group of this version. Similarly, the high-correlation condition in the transfer block refers to the pairs that were in the high-correlation condition during the training blocks for the experimental group. Because the pattern of results in Versions 1 and 2 were different, we analyzed them separately.

Version 1. We first present the analysis of the experimental group. For this group, RTs in the training blocks were

Table 2

Mean Reaction Times (in Milliseconds) and Proportion of

Errors for the High- and Low-Correlation Conditions of Versions 1 and 2 of Experiment 1

\begin{tabular}{|c|c|c|c|c|c|c|c|c|}
\hline \multirow[b]{3}{*}{ Correlation } & \multicolumn{4}{|c|}{ Experimental Condition } & \multicolumn{4}{|c|}{ Control Condition } \\
\hline & \multicolumn{2}{|c|}{ Training } & \multicolumn{2}{|c|}{ Transfer } & \multicolumn{2}{|c|}{ Training } & \multicolumn{2}{|c|}{ Transfer } \\
\hline & $M$ & PE & $M$ & $\mathrm{PE}$ & $M$ & $\mathrm{PE}$ & $M$ & PE \\
\hline \multicolumn{9}{|c|}{ Version 1} \\
\hline High & 477 & .018 & 483 & .026 & 473 & .016 & 474 & .019 \\
\hline Low & 502 & .047 & 472 & .015 & 462 & .013 & 460 & .015 \\
\hline $\mathrm{CE}$ & 25 & & -11 & & -11 & & -14 & \\
\hline \multicolumn{9}{|c|}{ Version 2} \\
\hline High & 460 & .013 & 460 & .013 & 543 & .015 & 529 & .012 \\
\hline Low & 517 & .09 & 483 & .033 & 547 & .019 & 539 & .014 \\
\hline $\mathrm{CE}$ & 57 & & 23 & & 4 & & 10 & \\
\hline
\end{tabular}

Note - CE. correlational cuing effect. 
faster in the high-correlation than in the low-correlation condition. Surprisingly, however, the pattern of results was reversed in the transfer block. RTs were slower in the high-correlation than in the low-correlation condition. A 2 (type of block: training, transfer) $\times 2$ (correlation: high, low) analysis of variance (ANOVA) was performed. The two main effects were not statistically significant. The block $\times$ correlation interaction was significant $[F(1,14)=$ $47.4, p<.05]$. We therefore analyzed the training and transfer blocks separately. The correlation effect was significant for the training blocks $[F(1,14)=14.8, p<.05]$. This reflects the standard finding that subjects respond faster in the high-correlation condition. The correlation effect was significant for the transfer block as well $[F(1,14)=$ $8.2, p<.05]$. This finding, however, is in the opposite direction to that expected.

The results for the control group clarify this finding. As can be seen in Table 2, the results of the training and transfer blocks for this group were very similar, and both were similar to the results obtained in the transfer block of the experimental condition. A 2 (type of block) $\times 2$ (correlation) ANOVA for the control group revealed a main effect of correlation $[F(1,14)=34.7, p<.05]$. No other effect was statistically significant. The control group represents the baseline condition, because every target-flanker pair was presented equally often. The findings from this group suggest that it is not possible to treat different target-flanker pairs as equal prior to the experimental manipulation. Subjects respond to certain target-flanker pairs faster than to others. Specifically, subjects respond faster to a blue target when it is flanked by green distractors than when it is flanked by red distractors. Similarly, subjects are faster to yellow targets flanked by red distractors than to those flanked by green distractors.

These findings suggest that, whereas correlational cuing affected the performance in the training blocks, it did not affect performance in the transfer block. To verify this impression we performed a 2 (group: experimental, control) $\times 2$ (correlation: high, low) ANOVA for the training blocks and for the transfer block. The main effect of the correlation was significant for the training blocks $[F(1,28)=4.6, p<.05]$. More important, the group $\times$ correlation interaction was significant for this group $[F(1,28)=26.5, p<.05]$, suggesting that the experimental group took advantage of the experimental correlation during the training. The main effect of correlation was significant for the transfer block as well $[F(1,28)=24.8, p<$ $.05]$. The group $\times$ correlation interaction, however, did not approach statistical significance in this block $[F(1,28)<1]$. This finding indicates that the CE disappears when the correlations are removed.

Version 2. As in Version 1, RTs for the experimental group in the training blocks were faster in the highcorrelation than in the low-correlation condition. Unlike Version 1, however, the pattern of results remained the same in the transfer block, although the magnitude of the effect was smaller. A 2 (type of block: training, transfer) $\times 2$ (correlation: high, low) ANOVA revealed that the main effect of correlation was significant $[F(1,14)=45.2$, $p<.05]$. The block $\times$ correlation interaction was significant as well $[F(1,14)=48, p<.05]$. Because of the interaction, we analyzed the training and transfer blocks separately. The correlation effect was significant for the training blocks $[F(1,14)=64.9, p<.05]$. The correlation effect was significant in the transfer block as well $[F(1,14)=$ $14.3, p<.05]$.

The results of Version 2 fit nicely with the standard interpretation of correlational cuing (e.g., Miller, 1987). The subjects appeared to learn the correlation between the targets and the flankers, and this learning was still observed in the transfer block. However, as is demonstrated in Version 1 , the effect of the transfer block could be due to a preexisting tendency to respond faster to certain targetflanker pairs than to others. Thus, the key comparison is between the experimental and the control groups. As can be seen in Table 2, the subjects in the control group responded faster to the high-correlation pairs as well, although this effect is quite small. A 2 (type of block) $\times 2$ (correlation) ANOVA for the control group revealed that the main effect of correlation approached significance $[F(1,14)=4.35, p<.06]$. No other effect was statistically significant.

To compare the performances of the experimental and control groups, we performed a 2 (group: experimental, . control) $\times 2$ (correlation: high, low) ANOVA for the training blocks and the transfer block. All the main effects were significant for the training blocks $[F(1,28)=5.1$ and $F(1,28)=62, p<.05$, for group and correlation, respectively]. Importantly, the group $\times$ correlation interaction was significant as well in these blocks $[F(1,28)=47, p<$ $.05]$, indicating that the experimental group used the experimental correlation during the training. The two main effects of group and correlation were significant in the transfer blocks too $[F(1,28)=9.2$ and $F(1,28)=17.5$, respectively, $p<.05$ for both effects]. However, as in Version 1 , the group $\times$ correlation interaction did not reach statistical significance in the transfer block $[F(1,28)=2.31$, $p>.05]$. This finding indicates that the advantage shown by the experimental group for the high-correlation pairs in the transfer block may be ascribed to a preexisting tendency to respond faster to certain target-flanker pairs and is not caused by learning of the correlations. Nevertheless, unlike Version 1, there was a (nonsignificant) tendency for a small amount of residual learning in the experimental group during the transfer block. We now turn to analyses that directly compare Versions 1 and 2, to clarify this issue.

Comparison of Versions 1 and 2. We first compare the transfer blocks for the experimental groups of Versions 1 and 2. The control groups showed that two of the four target-flanker pairs (blue-green and yellow-red) were easier than the other two pairs (blue-red and yellowgreen). The training of the experimental group in Version 2 reinforced this tendency, but the training in Version 1 worked against this preexisting tendency. Thus, to the extent that learning took place during training, we should find a lesser tendency to respond faster to the easy 
Table 3

A Breakdown of the Results in Different Quarters of the Trials for the Transfer Block in Experiment 1

\begin{tabular}{cccccc} 
& \multicolumn{3}{c}{ Correlation } \\
\cline { 2 - 3 } & \multicolumn{2}{c}{ Version 1 } & & \multicolumn{2}{c}{ Version 2 } \\
\cline { 2 - 3 } Quarter & High & Low & & High & Low \\
\hline Ist & 485 & 471 & & 461 & 480 \\
2nd & 492 & 479 & & 460 & 499 \\
3rd & 486 & 479 & & 477 & 489 \\
4th & 500 & 478 & 479 & 494 \\
\hline
\end{tabular}

pairs in the transfer block of Version 1 than in that of Version 2 . We ran a 2 (version: 1,2$) \times 2$ (preexisting tendency: easy pairs, difficult pairs) to test this prediction. The main effect of preexisting tendency was significant $[F(1,28)=22.5, p<.05]$, confirming the finding from the control groups that subjects respond faster to the two easy pairs. The version $X$ preexisting tendency interaction did not approach significance $[F(1,28)=1.4, p>$ .24], indicating once again that the training for the experimental groups did not affect performance in the transfer blocks. Nevertheless, there was a small difference $(12 \mathrm{msec})$ in the main effect of preexisting tendency between the two versions. Thus, it is still possible that there was a small residual learning effect in Version 2 and that a higher statistical power than that in our experiment would have revealed this residual learning.

Examination of Table 2 also reveals that the $\mathrm{CE}$ for the experimental groups during the training blocks appears to be larger in Version 2 than in Version 1. A 2 (version: 1,2) $\times 2$ (correlation: high, low) confirmed this examination. The main effect of correlation was significant $[F(1,28)=$ $72.3, p<.05]$. More important, the version $\times$ correlation interaction was also significant $[F(1,28)=10.3, p<.05]$, suggesting that the CE was larger in Version 2.

What is the reason for the difference between the two versions? One possible interpretation is that the $C E$ is superimposed on the preexisting differences between the various target-flanker pairs. As was shown in the two control groups, although there was no contingency between the targets and the flankers, the identity of the irrelevant flankers affected the response. The CE may be additive with this effect. Indeed, as can be seen in Table 2, the difference in $\mathrm{CE}$ between the training and the transfer blocks is similar in Version 1 (36 msec) and Version 2 (34 msec).

Alternatively, there may be another difference between Versions 1 and 2 in residual learning in the transfer block. Whereas there is no indication of such residual learning in Version 1, there is a tendency for a small amount of residual learning in Version 2. Thus, it is possible that most of the CE is common to Versions 1 and 2 but that a small amount of additional CE (presumably owing to a different source) is present only in Version 2, and this leads to a bigger $\mathrm{CE}$ in this condition.

Finally, the analyses of the transfer block were done by averaging across the entire block (of 160 trials). These analyses did not show any indication of learning of the correlations. It is possible, however, that the subjects learned the correlations, but "unlearned" these same correlations during the transfer block. This hypothesis predicts that subjects would show the CE in the early part of the transfer block and stop showing it later. To examine this prediction, we analyzed the first, second, third, and fourth quarters of the transfer blocks (consisting of 40 trials each) for the experimental groups of Versions 1 and 2 separately; the results are shown in Table 3. It is apparent that the magnitude of the CE was quite similar in all the quarters. Separate 2 (correlation) $\times 4$ (block quarter) analyses confirmed this observation. The correlation effect was significant in both versions $[F(1,14)=5.4$ for Version 1 and $F(1,14)=13$ for Version $2, p<.05$ for both versions]. Note that the correlation effect for Version 1 is in the opposite direction to what would be expected if learning took place during the correlation manipulation. Neither the block quarter nor the block quarter $\times$ correlation effect approached significance for either version $[F(3,42)<$ 1 in all these cases].

\section{EXPERIMENT 2}

Miller (1987) hypothesized that much of the CE is due to associations formed between flankers and assigned responses. The lack of transfer in Experiment 1 suggests that the subjects did not form lasting associations between the flankers and the responses, as was suggested by Miller (1987). Experiment 2 attempted to examine whether the learning Miller (1987) postulates does take place during the training itself. Miller's (1987) hypothesis predicts that considerably less learning will take place if two targets that require two different responses are correlated with the same flanker, because the flanker cannot be associated with the assigned response. The present experiment tested this prediction.

The task in Experiment 2 was similar to that of Experiment 1, with two major changes. First, the two targets were paired in a similar manner with both flankers. That is, one of the flankers appeared on $90 \%$ of the trials with both targets, and the other flanker appeared on $10 \%$ of the trials with both targets. Second, the subjects did not receive a transfer block.

\section{Method}

Subjects. Thirty subjects from the Hebrew University were tested in one session, either as part of their course requirements or for a payment of approximately $\$ 5$ per session. Fifteen of the subjects were tested in one version, and the remaining 15 participated in the second version.

Apparatus. The apparatus was the same as that in Experiment 1. Stimuli and Design. The stimuli presented in each display were taken from the same set of stimuli as were those in Experiment 1. However, both targets appeared with the same flanker on $90 \%$ of the trials and with the other flanker on $10 \%$ of the trials. As in Experiment 1 , we used two versions. In Version 1 , both targets appeared with the red flanker on $90 \%$ of the trial and with the green flanker on the 
remaining trials. The pairing was reversed in Version 2; both targets appeared with the green flanker on $90 \%$ of the trials and with the red one on $10 \%$ of the trials.

As in Experiment 1, each block consisted of 160 trials. Within each block, the two high-correlation pairs appeared in 72 trials each, and the two low-correlation pairs appeared in 8 trials each. The order of trials within each block was determined randomly. Each subject first received a practice block, followed by four experimental blocks.

Procedure. The procedure was the same as that in Experiment 1.

\section{Results and Discussion}

Table 4 presents the mean RTs and proportion of errors in the experimental blocks for the two versions. As in Experiment 1 , we analyzed the results separately for the two versions. The effect of correlational cuing was significant for the first version $[F(1,14)=82.5, p<.05]$. The same was true for the second version $[F(1,14)=34.9, p<.05]$. It is apparent from these results that a robust CE took place, even though it was not possible to associate the flankers with assigned responses.

Is the CE displayed in the present experiment similar to that displayed in Experiment 1 ? This question is interesting because, in the present experiment, the flankers could not be associated with assigned responses, whereas in Experiment 1 they could be. Thus, differences in CE between the two experiments may provide important clues for the contribution of flanker-response association to the CE displayed in Experiment 1. Because the results of Experiment 1 showed that there are differences among the various target-flanker pairs, we compared the magnitude of the CE in the two experiments separately for each of the four pairs (see Table 5).

As can be seen in Table 5, the CE in two of the pairs (blue-red and yellow-green) was larger in the present experiment, whereas the $\mathrm{CE}$ for the other two pairs was larger in Experiment 1. Interestingly, the former pairs were used for the high-correlation condition in Version 1, whereas the latter were used for the high-correlation pairs in Version 2 of Experiment 1. Moreover, in Experiment 1, there was no indication of residual learning during the transfer block of Version 1, but there was some (albeit small and statistically nonsignificant) residual learning in Version 2. We performed a 2 (experiment: 1,2$) \times 2$ (correlation: high, low) mixed ANOVA for each of the four pairs. An experiment $\times$ correlation interaction would indicate that the $C E$ was different in the two experiments. This interaction was not statistically significant for three of the pairs: blue-red $[F(1,28)=2, p>.16]$, yellow-red $[F(1,28)=$ $1.56, p>.022]$, and yellow-green $[F(1,28)<1]$. The inter-

Table 4

Mean Reaction Times (in Milliseconds) and Proportion of Errors for the High-Correlation and Low-Correlation Conditions in Experiment 2

\begin{tabular}{|c|c|c|c|c|}
\hline \multirow[b]{2}{*}{ Correlation } & \multicolumn{2}{|c|}{ Version 1} & \multicolumn{2}{|c|}{ Version 2} \\
\hline & $M$ & $\mathrm{PE}$ & $M$ & $\mathrm{PE}$ \\
\hline High & 472 & .016 & 509 & .013 \\
\hline Low & 504 & .027 & 545 & .025 \\
\hline
\end{tabular}

Table 5

\begin{tabular}{|c|c|c|c|c|}
\hline \multirow[b]{2}{*}{ Correlation } & \multicolumn{4}{|c|}{ High Pair } \\
\hline & Blue-Green & Blue-Red & Yellow-Green & Yellow-Red \\
\hline \multicolumn{5}{|c|}{ Experiment 1} \\
\hline High & 467 & 486 & 467 & 453 \\
\hline Low & 529 & 504 & 500 & 505 \\
\hline $\mathrm{CE}$ & 62 & 18 & 33 & 52 \\
\hline \multicolumn{5}{|c|}{ Experiment 2} \\
\hline High & 510 & 482 & 508 & 464 \\
\hline Low & 542 & 502 & 546 & 506 \\
\hline CE & 32 & 20 & 38 & 42 \\
\hline
\end{tabular}

Note - The high pair is the pair that was presented on $90 \%$ of the trials. $\mathrm{CE}$, correlational cuing effect.

action was significant, however, for the blue-green pair $[F(1,28)=15.8, p<.05]$.

These results suggest that the correlation between the flankers and the responses is not a major source for the CE. The findings indicate that, with the exception of the blue-green pair, the $C E$ is not affected when associations between the flankers and the assigned responses are prevented from forming.

\section{EXPERIMENT 3}

Given the lack of $\mathrm{CE}$ in the transfer block of Experiment 1 , it appears to be a more local effect confined to processes occurring during the performance of the training blocks. Experiment 2 demonstrated that associating the flankers with correlated responses during training also contributes very little. What then is the nature of these local processes?

One class of possible explanations focuses on fallout from the correlational cuing design. Typical correlational cuing paradigms lead to large differences among the various displays. In particular, the high-correlation displays appear more often than the low-correlation ones. For example, in our experiments, the two high-correlation displays appeared nine times as often as the low-correlation ones. This frequency difference may have several consequences. For example, high-correlation displays may become more familiar, and this familiarity could facilitate the response. In addition, subjects may anticipate the appearance of such displays and respond faster to them.

In addition to these direct frequency effects, there may be indirect effects as well. In particular, differences in frequency also lead to differences in contingencies between successive trials. The importance of several different contingencies has been documented by Mordkoff and Yantis (1991) in a divided-attention paradigm. Moreover, Mordkoff (1996) showed that contingencies may affect the flanker task as well. However, Mordkoff also demonstrated that the flanker congruency effect can be observed even when all contingencies are eliminated. No study to date has examined the importance of contingencies in the 
correlational cuing paradigm. The purpose of the present experiment was to demonstrate that one such contingency, which we call repetition priming contingency, is an important factor in determining the $\mathrm{CE}$.

In Experiment 3, we examined the hypothesis that two successive presentations of the same display cause a faster response to the second display. As was mentioned earlier, many studies have shown the existence of repetition priming (e.g., Bertelson, 1963; Kornblum, 1969). However, these studies have focused on target priming rather than display priming. Our hypothesis is that repetition of the display (which includes the target and the irrelevant distractors) leads to a faster response to the repeated display. The existence of this type of repetition priming can account for the CE in our paradigm because, owing to the correlation manipulation in our experiment, the probability of two successive identical displays was much higher for the high-correlation condition than for the low-correlation condition. It is thus possible that differences in repetition priming contingencies between high- and low-correlation conditions caused some or even all of the CE.

\section{Method}

Subjects. Fifteen subjects from the Hebrew University were tested in one session, either as part of their course requirements or for a payment of approximately $\$ 5$ per session.

Apparatus. The apparatus was the same as that in Experiment 1.

Stimuli and Design. The stimuli presented in each display were taken from the same sets of target and flanker stimuli as were those in Experiment 1. Each target appeared equally often with the two flankers, with a minor exception noted below. In addition, we manipulated the contingency between successive trials. Each of the four displays was followed equally often by each of the four displays, and we did not allow a run of more than two identical trials.

To create 160 repetitions of the trials, each block consisted of 161 trials. To achieve a balanced repetition among the four displays, the display that appeared in the first trial of the block (randomly chosen among the four displays) also appeared in the last trial of the block. This display thus appeared on 41 trials in the block, whereas the remaining displays appeared in 40 trials each. Each display was followed 10 times by each of the four displays. Within these constraints, the order of trials in the block was determined randomly. Each subject first received a practice block, followed by four experimental blocks.

Procedure. The procedure was the same as that in Experiment 1.

\section{Results and Discussion}

There were four main conditions in this experiment, involving different relations between the display in the trial to which the subjects were asked to respond and the display in the preceding trial. The first condition, which we called same target same flankers (STSF), involves a repetition of both target and flankers. The second condition, same target different flankers (STDF), is a repetition of the target and a change of the flankers. The third condition, different target same flankers (DTSF), is a change of the target and a repetition of the flankers. The fourth condition, different target different flankers (DTDF), is a change of both target and flankers. Because the number of trials for each subject in each condition was relatively small, we col-
Table 6

Mean Reaction Times (in Milliseconds) and Proportion of Errors for the Four Main Conditions in Experiment 3

\begin{tabular}{ccc}
\hline Condition & RT & Proportion of Errors \\
\hline STSF & 481 & .017 \\
STDF & 499 & .032 \\
DTSF & 510 & .029 \\
DTDF & 505 & .032 \\
\hline
\end{tabular}

Note-STSF, same target, same flankers; STDF, same target, different flankers; DTSF, different target, same flankers; DTDF, different target, different flankers.

lapsed the results across the four pairs; they are shown in Table 6.

As can be seen in Table 6, the subjects were fastest in the repetition (STSF) condition. There were also smaller differences among the other three conditions. An ANOVA revealed that the difference among the four conditions was significant $[F(3,42)=8.5, p<.05]$. The most interesting comparison is that between the STSF and the STDF conditions, because the target was repeated in both conditions, satisfying the typical definition of repetition priming, whereas the flankers were repeated only in the former. A planned contrast revealed a significant difference between the two conditions $[F(1,14)=17.04, p<.05]$. Another interesting comparison is that between the response for the STDF condition and those for the DTSF and DTDF conditions, because the target was repeated in the former and not in the latter. Although the mean RT was faster for the STDF condition, the contrast between this condition and the two other conditions was not significant $[F(1,14)=$ $1.16, p>.3] .^{1}$

These results demonstrate the existence of display repetition priming. As far as we know, this is the first demonstration of such priming. Several recent reports documented other types of novel priming effects (Maljkovic \& Nakayama, 1994; Robertson, in press). Note that we cannot be sure whether the priming effect observed in our study is due to positive priming of the repeated display or to slowing the response for the nonrepeated display. More experiments are required to settle this issue. More important for our purpose, the results suggest one mechanism that contributes to the $\mathrm{CE}$. In a typical correlational cuing paradigm used in Experiments 1 and 2, the proportion of trials in which there is repetition of the display is much higher in the high-correlation than in the low-correlation condition. As the results of the present experiment demonstrate, RT is shorter in these trials and, consequently, the mean RT for the high-correlation condition is shorter as well. Although these findings account for some of the CE, they do not appear to account for all the effect. The size of the effect $(18 \mathrm{msec})$ is smaller than that obtained in Experiments 1 and 2 . As was mentioned above, other local factors (e.g., display familiarity) may also contribute to this effect. More research is required to uncover these additional factors. 
The results obtained in the first three experiments appear straightforward: The CE is primarily and perhaps entirely due to processes that occur during training. These processes do not appear to involve association between flankers and responses, but are caused at least partly by repetition priming of the display. As was mentioned in the introduction, however, the results obtained by Miller (1987) appear to contradict our results. The goal of the remaining experiments was to find out the reasons for this difference.

A notable difference between our method and Miller's (1987) is that he used letters as stimuli, whereas we used colors. There are many differences between colors and letters. One difference is that letters are composed of shape features (e.g., line orientations, curvature), whereas colors are part of the surface features. A number of researchers (e.g., Biederman, 1987) have suggested that there may be fundamental differences between these two types of features. The next experiment examined correlational cuing with an orientation task.

\section{EXPERIMENT 4}

This experiment is very similar to Experiment 1 , except that the task required discrimination among orientations rather than colors. Two line orientations (right diagonal and left diagonal) served as targets, and two other line orientations (horizontal and vertical) served as flankers. As in Experiment 1, we used two versions. In Version 1, the right diagonal line appeared in high correlation with the horizontal line, and the left diagonal target appeared in high correlation with the vertical line. This correlation was reversed in Version 2. Following four training blocks, the subjects were shifted to a transfer block in which there was no correlation between the targets and the flankers.

\section{Method}

Subjects. Thirty subjects from the Hebrew University were tested in one session, either as part of their course requirements or for a payment of approximately $\$ 5$ per session. Fifteen subjects participated in Version 1, and the remaining subjects in Version 2.

Apparatus. The apparatus was the same as that in the previous experiments.

Stimuli and Design. The design and stimuli were identical to those of Experiment 1, except for the following. We did not use control groups in this experiment. The stimuli were achromatic lines. The two targets were diagonal lines tilted $45^{\circ}$ to either the right (Response 1) or the left (Response 2). The two flankers were vertical and horizontal lines. The size of the stimuli was identical to that used

Table 7

Mean Reaction Times (in Milliseconds) and Proportion of Errors for the High- and Low-Correlation Conditions of Versions 1 and 2 in Experiment 4

\begin{tabular}{|c|c|c|c|c|c|c|c|c|}
\hline \multirow[b]{3}{*}{ Correlation } & \multicolumn{4}{|c|}{ Version 1} & \multicolumn{4}{|c|}{ Version 2} \\
\hline & \multicolumn{2}{|c|}{ Training } & \multicolumn{2}{|c|}{ Transfer } & \multicolumn{2}{|c|}{ Training } & \multicolumn{2}{|c|}{ Transfer } \\
\hline & $M$ & $\mathrm{PE}$ & $M$ & $\overline{P E}$ & $M$ & PE & $M$ & $P E$ \\
\hline High & 482 & .01 & 473 & .01 & 455 & .01 & 456 & .01 \\
\hline Low & 494 & .02 & 476 & .01 & 464 & .01 & 455 & .01 \\
\hline $\mathrm{CE}$ & 12 & & 3 & & 9 & & -1 & \\
\hline
\end{tabular}

Note-CE, correlational cuing effect. in the previous experiments. In Version 1, during the four training blocks, the right diagonal target appeared with the horizontal flankers on $90 \%$ of the trials and with the vertical flankers on $10 \%$ of the trials. The correlation was reversed for the left diagonal target. The pairing of the two targets and the two flankers was reversed in the second version. As before, the correlation was eliminated in the transfer block for all versions.

Procedure. The procedure was the same as that in the previous experiments.

\section{Results and Discussion}

The results of the two versions are shown in Table 7. Because they were quite similar, we analyzed the two versions together. To make sure that this assumption was justified, we performed a 2 (version: 1,2$) \times 2$ (correlation) $\times 2$ (type of block: training, transfer) ANOVA. The main effect of version was not significant, nor did it interact with the other main effects, supporting our assumption that the CEs were similar in the two versions. The main effect of correlation was significant $[F(1,28)=26, p<.05]$. However, the block $\times$ correlation interaction was significant as well $[F(1,28)=4.7, p<.05]$. We therefore analyzed the training and transfer blocks separately. The correlation was significant for the training blocks $[F(1,28)=$ $19.4, p<.05]$. This result demonstrates that the subjects took advantage of the correlations in the training blocks. In contrast, the correlation did not approach significance for the transfer block $[F(1,28)<1]$.

These findings replicate the main results obtained with color stimuli. Subjects show the $C E$ when the correlations exist. However, this effect disappears when the correlations are eliminated, indicating that the $C E$ is a local effect confined to processes that occur while the task is being performed with the manipulated correlations. Note that the CE is smaller than that observed for color stimuli. We do not know the reason for this observed difference. Recall that, in Experiments 1 and 2, we found differences in the magnitude of the correlational cuing effect between different pairs. It appears that the nature of the stimuli affect the size of this effect.

\section{EXPERIMENT 5}

The results of Experiment 4 showed that the difference between our findings and Miller's (1987) are not due to differences between surface features and shape features. Miller (1987) used letters. Letters are complex entities that, in all likelihood, involve conjunctions of simpler shape features, such as orientation and curvature (see, e.g., Treisman \& Gelade, 1980; see Wolfe \& Bennett, 1997, for a recent discussion). One possible source of the differential results between the two studies is related to the complexity of letters. In particular, the similarity relations among letters could be a factor in the CE.

Similarity between target and flankers has been shown to play a major role in a variety of paradigms (e.g., Duncan \& Humphreys, 1989). The influence of similarity between target and flankers on the congruency effect in a letter discrimination task has also been documented in the 
original flanker study (B. A. Eriksen \& C. W. Eriksen, 1974). However, we are not aware of a similar study with the correlational cuing paradigm. Miller (1987) sampled letters randomly for each subject in his experiments. Thus, it is not possible to estimate the effect of similarity in his experiments. The purpose of Experiment 5 was to examine this issue.

Unfortunately, the similarity metric among letters is not known. Although there have been a number of attempts to estimate the similarity structure among letters (e.g., Gibson, 1969; Townsend, 1971), such estimates are often derived from a particular task and may not be generalizable to other tasks. To circumvent this problem, we chose several different sets of letters on the basis of informal estimates of their similarity. Our goal was simply to demonstrate empirically that the similarity between targets and flankers in a letter discrimination task is an important factor in the correlational cuing paradigm.

\section{Method}

Subjects. Sixty subjects from the Hebrew University were tested in one session, either as part of their course requirements or for a payment of approximately $\$ 5$ per session. Four versions of a letter task were used, and 15 subjects were assigned to each version.

Apparatus. The apparatus was the same as that in the previous experiments.

Stimuli and Design. The design was similar to that of Experiment 4 . We used letters instead of line orientations. All the letters were achromatic and subtended $0.4^{\circ}$ of visual angle in width and $0.5^{\circ}$ in height. The center-to-center distance between the target and the flankers was approximately $0.52^{\circ}$ of visual angle. ${ }^{2}$ The targets in all the versions were the letters $S$ (Response 1 ) and $F$ (Response 2). The flankers in the first two versions were the letters $O$ and $X$. We assumed that $S$ is more similar to $O$ than to $X$ and that $F$ is more similar to $X$ than to $O$. In the first version, the target $S$ was correlated with the flanker $\mathrm{O}$, and the target $\mathrm{F}$ was correlated with $\mathrm{X}$. Thus, in this version, the target was more similar to its high-correlation flanker. The correlation was reversed for the second version. Thus, in this version, the target was more similar to its low-correlation flanker. In Versions 3 and 4, the flankers were the letters $C$ and $T$. Again, we assumed that $S$ is more similar to $C$ than to $T$ and that $F$ is more similar to $T$ than to $C$. In Version 3, the targets were correlated with their more similar flankers (i.e., $S$ with $C$ and $F$ with $T$ ), with the reverse situation for Version 4 . As before, the correlations were eliminated in the transfer block.

Procedure. The procedure was the same as that in previous experiments.

Table 8

Mean Reaction Times (in Milliseconds) and Proportion of Errors for the Individual Target-Flanker Pairs Within the HighCorrelation and Low-Correlation Conditions in Experiment 5

\begin{tabular}{|c|c|c|c|c|c|c|c|c|}
\hline \multirow[b]{2}{*}{ Correlation } & \multicolumn{2}{|c|}{ Version 1} & \multicolumn{2}{|c|}{ Version 2} & \multicolumn{2}{|c|}{ Version 3} & \multicolumn{2}{|c|}{ Version 4} \\
\hline & $M$ & $\mathrm{PE}$ & $M$ & $\mathrm{PE}$ & $M$ & $\mathrm{PE}$ & $M$ & $\mathrm{PE}$ \\
\hline \multicolumn{9}{|c|}{ Training Blocks } \\
\hline High & 459 & .01 & 449 & .02 & 467 & .02 & 472 & .02 \\
\hline Low & 494 & .05 & 472 & .03 & 512 & .08 & 488 & .03 \\
\hline $\mathrm{CE}$ & 35 & & 23 & & 45 & & 16 & \\
\hline \multicolumn{9}{|c|}{ Transfer Blocks } \\
\hline High & 471 & .01 & 448 & .03 & 473 & .03 & 477 & .03 \\
\hline Low & 482 & .02 & 440 & .01 & 511 & .05 & 466 & .02 \\
\hline $\mathrm{CE}$ & 11 & & -8 & & 38 & & -11 & \\
\hline
\end{tabular}

Note-CE, correlational cuing effect.

\section{Results and Discussion}

The results of the four versions are shown in Table 8. It is apparent that the results of the two versions in which the targets were correlated with their more similar flanker (Versions 1 and 3 ) are different from those of the two versions in which the targets were correlated with their less similar flankers. First, the CEs during training were larger for the former than for the latter. Second, a different pattern of transfer emerged for Versions 1 and 3 and for Versions 2 and 4 . Whereas a weak negative transfer was observed in Versions 2 and 4, a positive transfer emerged in Versions 1 and 3. This transfer is particularly clear in Version 3.

To verify these impressions, we conducted separate 2 (correlation) $\times 2$ (block) ANOVAs for each of the four versions. For Version 1, the main effect of correlation was significant $[F(1,14)=23.3, p<.05]$, as was the correlation $\times$ block interaction $[F(1,14)=8.5, p<.05]$. Separate contrasts showed that the correlation was significant in both training and transfer $[F(1,14)=21$ and $F(1,14)=$ 5.1 , respectively, $\mathrm{p}<.05$ for both]. For Version 3 , the main effect of correlation was significant $[F(1,14)=26, p<$ $.05]$. Interestingly, the correlation $\times$ block interaction was not significant $[F(1,14)=3.2, p>.09]$, indicating that the $\mathrm{CE}$ was similar in the transfer block. In general, these findings show that a marked CE occurred in the transfer block. Moreover, at least statistically, this effect was not significantly reduced in Version 3, even when the correlation was eliminated in the transfer block.

The ANOVA of Version 2 revealed that the main effect of correlation was not significant $[F(1,14)=3.65, p>$ $.07]$, but the correlation $\times$ block interaction was significant $[F(1,14)=17.8, p<.05]$. Separate contrasts showed that the effect of correlation was significant for the training blocks $[F(1,14)=17.9, p<.05]$. The effect was not significant, however, for the transfer block $[F(1,14)=$ $2.5, p>.13$ ]. A similar (although not identical) pattern was observed in Version 4 . The only significant effect was that of the correlation $\times$ block interaction $[F(1,14)=20.9, p<$ $.05]$. Separate contrasts revealed that the correlation effect was significant in the training blocks $[F(1,14)=8.4, p<$ $.05]$. The correlation effect was significant in the transfer block as well $[F(1,14)=5.7, p<.05]$. Note, however, that the correlation effect in the transfer block is in the opposite direction to that expected by learning of the correlations during the training blocks. Thus, there is no indication of transfer of learning in Versions 2 and 4.

Several observations are apparent from these results. First, the CE is observed during training in all the versions, but its magnitude depends on the degree of physical similarity between the targets and the flankers. Second, when the correlation is eliminated, the effect still exists for highcorrelation flankers that are similar to the targets. However, the effect disappears for high-correlation flankers that are dissimilar to the target. These results, then, clearly demonstrate that the degree of target-flanker similarity is a major factor in the correlational cuing paradigm with a letter discrimination task.

Our goal in this experiment was to start bridging the gap between our findings and those obtained by Miller 
(1987). The present experiment provides clear clues toward attaining this goal. For some high-correlation targetflanker pairs (e.g., those used in Versions 1 and 3), the pattern of results is compatible with that obtained by Miller (1987). However, for other combinations of target-flanker pairs, we replicated the pattern of results obtained in our earlier experiments. Can similar high-correlation targetflanker pairs (as in Versions 1 and 3) lead to the pattern of results obtained by Miller (1987)? In Experiment 6, we examined this issue.

\section{EXPERIMENT 6}

Experiment 5 revealed that it is possible to observe transfer of CE for similar target-flanker pairs. The transfer in our previous experiments was examined by eliminating the correlations. The task, however, remained the same, and there was no change in the sets of target and flanker stimuli. As mentioned earlier, Miller (1987, Experiment 3 ) demonstrated a more impressive type of transfer. Subjects were first trained on a typical correlational cuing task. In a subsequent block, the task was changed. The subjects were asked to respond to the stimuli that had served as flankers during the training blocks. Some of the targets in this transfer block were assigned a response to which they had been correlated during the training blocks (hereafter, the consistent targets), whereas other targets (hereafter, the inconsistent targets) were assigned a response that had been negatively correlated with them during the training blocks. Despite the change in the task, the effect of training was still observed in the transfer block. The subjects were faster when responding to the consistent targets than to the inconsistent targets.

Obtaining this type of transfer is more impressive than obtaining transfer in the method used in our previous experiments. The association of flankers with the response is implicit (i.e., the subjects are never asked to form such an association), whereas the association of targets with responses is explicit. A study by Cohen, Ivry, Rafal, and Kohn (1995) indicates that there is a difference between these two types of response association. Cohen et al. tested patients with neglect. Neglect is caused by a posterior brain lesion (typically around the temporal-parietal junction), which leads to a deficit in responding to stimuli that appear contralateral to the side of the lesion. In one task, the patients were asked to respond to a central target while a single flanker appeared on either the ipsilateral or contralateral side. The flanker could be congruent, incongruent, or neutral. Cohen et al. found approximately the same congruency effect for flankers on both sides. In another task, the same stimuli were used, but the patients were asked to ignore the central stimulus and respond to the peripheral stimulus (which was again presented either ipsilaterally or contralaterally). The patients were much slower when the target appeared contralateral to the side of the lesion. These findings demonstrate an impairment in explicit responses (as demonstrated in the second task) in conjunction with intact implicit response association (as demonstrated by the congruency effect in the first task). Put differently, forming implicit response associations does not imply that explicit response associations are achieved as well. Yet, Miller (1987) found a transfer from implicit to explicit response associations in the correlational cuing paradigm. We therefore wanted to verify that we could also obtain this transfer with the more similar high-correlation target-flanker pairs of Experiment 5.

\section{Method}

Subjects. Twenty-four subjects from the Hebrew University were tested in one session, either as part of their course requirements or for a payment of approximately $\$ 5$ per session. Two versions, with 12 subjects each, were used.

Apparatus. The apparatus was the same as that in previous experiments.

Stimuli and Design. The stimuli were the same as those in Experiment 5 . All the subjects received the same training. The targets during the training blocks were the letters $\mathrm{S}$ (Response 1 ) and F (Response 2). Unlike the procedure in the previous experiments, we used two high-correlation and two low-correlation flankers (see Table $9 \mathrm{~A}$ for details). The target $\mathrm{S}$ appeared in high correlation with the flankers $\mathrm{C}$ and $\mathrm{O}$ and in low correlation with the flankers $\mathrm{T}$ and $X$. The target $\mathrm{F}$ appeared in high correlation with the flankers $\mathrm{T}$ and $\mathrm{X}$ and in low correlation with the flankers $\mathrm{C}$ and $\mathrm{O}$. Note that the target was visually more similar to the high-correlation flankers than to the low-correlation flankers. The task was changed in the transfer block. A single target letter appeared in the center of the screen. The target could be one of the four flankers in the training blocks. The subjects were required to make one response to two of these targets and a different response to the other two targets (see Table 9B). One of the two targets associated with each response was consistent with the correlation used in the training blocks, and the other was inconsistent with it. To counterbalance for possible interactions between the response and the target, we used two versions (see Table 9B). A positive transfer in this design should lead to faster mean RTs for the consistent than for the inconsistent targets.

The subjects were first tested on a practice block, followed by four training blocks of 160 trials each, in which the correlation of the flankers was manipulated, as is shown in Table 9A. In these blocks, the four high-correlation target-flanker pairs appeared in 36 trials

Table 9A

The Degree of Correlation Used During the Training Blocks in Experiment 6

\begin{tabular}{cccc}
\hline Target & Flanker & Proportion of Trials & Correlation \\
\cline { 1 - 3 } S & O & 22.5 & high \\
S & C & 22.5 & high \\
S & X & 2.5 & low \\
S & T & 2.5 & low \\
F & X & 22.5 & high \\
F & T & 22.5 & high \\
F & O & 2.5 & low \\
F & C & 2.5 & low \\
\hline
\end{tabular}

Table 9B

Targets as a Function of Consistency During the Transfer Block

\begin{tabular}{cccccc} 
& \multicolumn{2}{c}{ Response 1 } & & \multicolumn{2}{c}{ Response 2 } \\
\cline { 2 - 3 } \cline { 5 - 6 } Version & Consistent & Inconsistent & & Consistent & Inconsistent \\
\hline 1 & $\mathrm{O}$ & $\mathrm{X}$ & & $\mathrm{T}$ & $\mathrm{C}$ \\
2 & $\mathrm{C}$ & $\mathrm{T}$ & $\mathrm{X}$ & $\mathrm{O}$ \\
\hline
\end{tabular}


each, and the four low-correlation pairs in 4 trials each. Within these constraints, the order of the trials in each block was random. The transfer block also consisted of 160 trials. Each of the four targets appeared in 40 trials. The order of the trials was random.

Procedure. The procedure was the same as that in the previous experiments.

\section{Results and Discussion}

As expected, a CE (of $30 \mathrm{msec}$ ) was observed during the training blocks. The mean RT was $453 \mathrm{msec}$ for the high-correlation trials and $483 \mathrm{msec}$ for the low-correlation trials. The difference between the two conditions was significant $[F(1,23)=70, p<.05]$. The main question of interest involved the transfer. The mean RT was $553 \mathrm{msec}$ for the consistent targets (proportion of errors, .03), and $568 \mathrm{msec}$ for the inconsistent target (proportion of errors, .06). The 15-msec difference was just significant in a onetailed $t$ test $[t(23)=1.94, p<.05]$. Thus, although the effect was fairly small, we also obtained transfer from implicit associations of flankers with responses to explicit associations of these stimuli with the responses.

\section{EXPERIMENT 7}

The previous experiments have several methodological properties that may limit their implications for other studies. With the exception of Experiment 6, only two flankers were used in each experiment. In addition, the high-correlation pairs were presented on $90 \%$ of the trials. Consequently, each of the high-correlation displays appeared quite often ( $45 \%$ of the trials in most experiments), and the probability of an immediate repetition of a high-probability display was fairly high (approximately .2). It is possible that these properties may have biased the subjects to attend to on-line local processes, such as frequency and repetition.

In addition, Experiments 5 and 6 used target-flanker pairs that were either similar (Versions 1 and 3 of Experiment 5 and both versions of Experiment 6) or dissimilar (Versions 2 and 4 of Experiment 5). By contrast, Miller (1987) had sampled the various target-flanker pairs randomly for each subject. This sampling could lead to situations in which some of the high-correlation target-flanker pairs are similar and some are not. Our more homogeneous design may have caused subjects to focus on the similarity structure between the targets and the flankers.

It is possible, therefore, that more complex situations (e.g., more flankers and a more complicated similarity structure) may induce subjects to examine more general correlational properties and that learning may be displayed in such situations. The final experiment was designed to examine this possibility. The targets in this experiment were the same as those in Experiments 5 and 6 . However, the set of flankers consisted of eight letters. Four of the flankers were correlated with one of the targets, and the other four flankers were correlated with the second target. The level of correlation was changed as well. The highcorrelation pairs were presented on $75 \%$ of the trials, and the low-correlation pairs were presented on the remaining $25 \%$ of the trials. As a result of these changes, each of the eight high-correlation displays appeared on less than $10 \%$ of the trials, and the probability of successive presentations of a high-probability display was substantially reduced as well (to approximately .009). This change ought to substantially reduce the influence of local processes, such as frequency and repetition priming, on the magnitude of the CE.

We also changed the similarity structure between the flankers and the targets. In Experiment 7, half of the highcorrelation flankers were similar to their correlated target, and the other half were dissimilar to it.

\section{Method}

Subjects. Forty-five subjects from the Hebrew University were tested in one session, either as part of their course requirements or for a payment of approximately $\$ 5$ per session. Fifteen of the subjects were tested in one experimental version, 15 other subjects participated in a second version, and 15 subjects participated in a control condition in which the flankers were not correlated with the targets.

Apparatus. The apparatus was the same as that in the previous experiments.

Stimuli and Design. The targets, as in Experiments 5 and 6, were the letters $\mathrm{S}$ and $\mathrm{F}$. The flankers set included the letters $\mathrm{O}, \mathrm{C}, \mathrm{Q}, \mathrm{U}$, $\mathrm{X}, \mathrm{T}, \mathrm{E}$, and $\mathrm{H}$. The size and luminance of the letters was the same as those in Experiments 5 and 6 . We assumed that the target $S$ is more similar to the first four flankers $(\mathrm{O}, \mathrm{C}, \mathrm{Q}$, and $\mathrm{U})$, all of them being curved, and that the target $F$ is more similar to the other four flankers $(X, T, E$, and $H)$, all of them being composed of straight lines. As before, we use this informal and rather crude measure of similarity to simply demonstrate empirically its importance.

We used two experimental versions and a control condition. In the experimental versions, four of the flankers appeared in high correlation with one target, and the other four flankers appeared in high correlation with the other target. Each target was similar to two of its high-correlation flankers and to two of its low-correlation flankers and dissimilar to the other two high-correlation and two lowcorrelation flankers. The pairing of the target and flankers was counterbalanced between the two experimental versions. The exact pairing of the two targets with the eight flankers in each of the two versions

Table 10

The Degree of Correlation Used During the Training Blocks in Experiment 7

\begin{tabular}{ccccc} 
& \multicolumn{2}{c}{ Flanker } & & \\
\cline { 2 - 3 } Target & Version 1 & Version 2 & Correlation & Similarity \\
\hline S & $\mathrm{O}$ & $\mathrm{Q}$ & high & high \\
$\mathrm{S}$ & $\mathrm{C}$ & $\mathrm{U}$ & high & high \\
$\mathrm{S}$ & $\mathrm{E}$ & $\mathrm{X}$ & high & low \\
$\mathrm{S}$ & $\mathrm{H}$ & $\mathrm{T}$ & high & low \\
$\mathrm{S}$ & $\mathrm{X}$ & $\mathrm{E}$ & low & low \\
$\mathrm{S}$ & $\mathrm{T}$ & $\mathrm{H}$ & low & low \\
$\mathrm{S}$ & $\mathrm{Q}$ & $\mathrm{O}$ & low & high \\
$\mathrm{S}$ & $\mathrm{U}$ & $\mathrm{C}$ & low & high \\
$\mathrm{F}$ & $\mathrm{X}$ & $\mathrm{E}$ & high & high \\
$\mathrm{F}$ & $\mathrm{T}$ & $\mathrm{H}$ & high & high \\
$\mathrm{F}$ & $\mathrm{Q}$ & $\mathrm{O}$ & high & low \\
$\mathrm{F}$ & $\mathrm{U}$ & $\mathrm{C}$ & high & low \\
$\mathrm{F}$ & $\mathrm{O}$ & $\mathrm{Q}$ & low & low \\
$\mathrm{F}$ & $\mathrm{C}$ & $\mathrm{U}$ & low & low \\
$\mathrm{F}$ & $\mathrm{E}$ & $\mathrm{X}$ & low & high \\
$\mathrm{F}$ & $\mathrm{H}$ & $\mathrm{T}$ & low & high \\
\hline
\end{tabular}

Note--Each target appeared with eight flankers, of which four were high-correlation and four were low-correlation flankers. Half of the flankers from each correlation set were similar to the target, and the other half were dissimilar 
is shown in Table 10. The targets were presented with high-correlation flankers on $75 \%$ of the trials and with low-correlation flankers on the remaining $25 \%$ of the trials. For each target, each high-correlation flanker appeared equally often (i.e., $18.75 \%$ of the trials), and each low-correlation flanker appeared equally often (i.e., $6.25 \%$ of the trials). Once again, following training with these correlations, we used a transfer block in which the correlations were eliminated, to evaluate the nature of the CE. Each training block included 160 trials in which each of the eight high-correlation displays (i.e., two targets with four high-correlation flankers) was presented on 15 trials, and each of the eight low-correlation displays was presented on 5 trials. The transfer block included 160 trials as well, 10 trials for each of the 16 possible displays. Each subject received a practice block followed by four training blocks and a transfer block.

As in Experiment 1, we also used a control condition. The subjects in this condition received the same amount of training as did those in the experimental conditions. However, the training blocks for this group were identical to the transfer block, and each target appeared equally often with the eight flankers throughout the experiment.

Procedure. The procedure was the same as that in previous experiments.

\section{Results and Discussion}

The results for the two experimental versions were very similar. Therefore, we collapsed the results across the two versions. Table 11, top part, presents the mean RTs and proportion of errors for the experimental group in the various conditions. The design used in this experiment enables a separate examination of similarity (assessed by the difference between the high-correlation similar and the high-correlation dissimilar conditions and by the difference between the low-correlation similar and the lowcorrelation dissimilar conditions) and correlation (assessed by the difference between high-correlation similar and lowcorrelation similar conditions, as well as the difference between the high-correlation dissimilar and low-correlation dissimilar conditions). In addition, we were interested in the comparison between performance in the training and that in the transfer blocks.

A 2 (similarity) $\times 2$ (correlation) $\times 2$ (type of block: training, transfer) ANOVA was performed to examine these factors. The only significant main effect was that of similarity $[F(1,29)=25, p<.05]$. The type of block $\times$ correlation $\times$ similarity triple interaction was significant as well $[F(1,29)=4.68, p<.05]$. No other main effect or interaction was significant. To examine the nature of the

Table 11

Mean Reaction Times (in Milliseconds) and Proportion of Errors for the Correlation and Similarity Conditions During Training and Transfer in Experiment 7

\begin{tabular}{|c|c|c|c|c|c|}
\hline \multirow[b]{2}{*}{ Correlation } & \multirow[b]{2}{*}{ Similarity } & \multicolumn{2}{|c|}{ Training } & \multicolumn{2}{|c|}{ Transfer } \\
\hline & & $M$ & $\mathrm{PE}$ & $M$ & $\mathrm{PE}$ \\
\hline \multicolumn{6}{|c|}{ Experimental Conditions } \\
\hline High & high & 419 & .02 & 416 & .02 \\
\hline Low & high & 424 & .02 & 422 & .02 \\
\hline High & low & 427 & .04 & 431 & .04 \\
\hline Low & low & 432 & .04 & 425 & .04 \\
\hline \multicolumn{6}{|c|}{ Control Conditions } \\
\hline & high & 433 & .02 & 431 & .02 \\
\hline & low & 442 & .03 & 439 & .02 \\
\hline
\end{tabular}

triple interaction, we performed separate 2 (similarity) $\times$ 2 (correlation) ANOVAs for the training and for the transfer blocks. For the training blocks, both main effects were significant $[F(1,29)=22.7$ and $F(1,29)=8.35$ for the similarity and correlation effects, respectively, $p<.05]$. The similarity $\times$ correlation interaction did not approach significance $[F(1,29)<1]$. For the transfer block, the main effect of similarity was significant $[F(1,29)=13.7, p<.05]$, as was the similarity $\times$ correlation interaction $[F(1,29)=$ $6.4, p<.05]$. The main effect of correlation did not approach significance $[F(1,29)<1]$.

These results fit nicely with those of Experiments 5 and 6. There is a clear effect of similarity (approximately $8 \mathrm{msec}$ in the present experiment) and a small effect of CE during training (approximately $5 \mathrm{msec}$ in the present experiment). The effect of similarity is still present in the transfer block, but the overall effect of correlation disappears. Instead, the CE is still observed for high-correlation similar pairs, but, in fact, is in the wrong direction for highcorrelation dissimilar pairs.

The effect of similarity can also be observed in the control condition, shown in Table 11, bottom part. Similar pairs were $9 \mathrm{msec}$ faster in the training block and $8 \mathrm{msec}$ faster in the transfer block. This difference is very similar in magnitude to that observed for the experimental group. A 2 (similarity) $\times 2$ (type of block) ANOVA was performed to verify these impressions. The main effect of similarity was significant $[F(1,14)=23, p<.05]$. No other effect approached significance.

Interestingly, the magnitude of the $\mathrm{CE}$ in the present experiment was much smaller than those observed in Experiments 5 and 6 . This difference is in accord with the claim that $\mathrm{CE}$ is primarily due to local on-line processes, such as repetition priming and frequency. As was noted earlier, the frequency of each display and the probability of successive presentations of the same display were much lower in the present experiment, and therefore, a smaller effect was to be expected.

\section{GENERAL DISCUSSION}

The results of the present study can be divided roughly into two parts. In the first part, we examined CEs with targets and flankers that were defined by simple properties of color and orientation. The findings from this section strongly indicate that there is very little transfer from training to situations in which no correlation exists. Put differently, the CE for color and orientation is not due to learning but is rather caused by processes that take place while the correlation manipulation is being carried out. The lack of transfer was documented in Experiment 1 (for color targets) and Experiment 4 (for orientation targets). Experiment 2 indicated that the local processes causing the CE do not involve the association of the flankers with assigned responses. Experiment 3 demonstrated that one of the processes that lead to the $\mathrm{CE}$ is repetition priming of the display. An additional finding, not directly related to the $\mathrm{CE}$, is that a lack of correlation does not imply (as 
is usually assumed) that all target-flanker pairs are equal. There are preexperimental differences between various target-flanker pairs that affect performance as well.

The second section examined CEs with targets and flankers being defined by letters. This set of experiments was carried out in an effort to bridge the gap between our results and those obtained by Miller (1987). The results from this set of experiments revealed that a major factor in the CE is the degree of similarity between the targets and the flankers. Experiment 5 showed that when the targets and flankers were dissimilar, the results paralleled those obtained with color and orientation-namely, the effect appeared to be confined to processes that occur during training. When the targets and flankers were similar, however, there was transfer to tasks in which no correlation was present. Moreover, Experiment 6 replicated the results of Miller and showed that transfer is obtained even when the task is changed and the flankers become the targets. Finally, Experiment 7, using a larger number of flankers, showed that when a mixture of similar and dissimilar target-flanker pairs are correlated, an overall CE can be observed during training. This effect, however, is observed for similar but not for dissimilar pairs, once the correlation manipulation is removed.

In the remainder of this discussion, we focus on two issues. First, we try to explain our results and those obtained by Miller (1987) within a single framework. This explanation is admittedly speculative but is compatible with the extant results. Second, we discuss possible implications of our study for other studies with the correlational cuing paradigm.

\section{Components of the Correlational Cuing Effect: Toward a Reconciliation}

We turn first to the discrepancy between our results and those of Miller (1987). Our results show that, for target and flankers defined by color and orientation and for dissimilar target-flanker pairs, the correlational cuing effect is not transferred to situations in which the correlations are eliminated. Transfer is observed, however, for similar targetflanker pairs. By contrast, Miller (1987) presented evidence indicating that correlational cuing leads to associations between the flankers and their correlated responses.

To account for these discrepant findings, we need to look carefully at Miller's (1987) method. Miller sampled the various target-flanker pairs randomly for each subject. A straightforward way to account for Miller's results is to assume that the probability of sampling similar highcorrelation target-flanker pairs (such as those used in Versions 1 and 3 of Experiment 5 ) is greater than that of sampling pairs such as those used in Versions 2 and 4. If so, averaging across subjects will lead to a pattern of results similar to that of Versions 1 and 3-namely, the pattern obtained by Miller. This pure sampling explanation provides a reasonable description of the findings of Experiment 5. However, it does not explain why we get the pattern of results for the similar target-flanker pairs (i.e., the findings with Versions 1 and 3).
In addition, there are at least two findings with letter targets that appear to be at odds with this pure sampling explanation. First, Miller (1987, Experiment 3) and Experiment 6 of the present study showed transfer from an implicit association of flankers and their correlated response to an explicit association. Second, Miller (1987, Experiment 2) asked subjects to make one response to the appearance of one of three target letters and a different response to the appearance of one of three other targets. Two targets belonging to the first response set were correlated with one flanker, and two targets belonging to the other response set were correlated with another flanker. The third target in each of the two response sets was not correlated with any of the flankers. Miller (1987) found that RTs for targets that were not correlated with flankers were faster (by $12 \mathrm{msec}$ ) when they were flanked by stimuli that were correlated with their assigned response (i.e., flankers that were correlated with the two targets that belonged to the same response set) than when flanked by stimuli that were correlated with the alternative response. These findings cannot be explained by a random sampling of the letters. What is the explanation for these results?

We begin with an observation, made by a number of studies (e.g., Prinzmetal, Presti, \& Posner, 1986; Treisman $\&$ Gelade, 1980), that letters are complex visual entities. Consequently, discrimination among letters can often be based on several different visual properties other than the whole letter. For example, discrimination between the letters $\mathrm{X}$ and $\mathrm{O}$ may be based on curved versus straight lines, presence versus absence of closure, presence versus $a b-$ sence of intersection, and so on. Thus, a correct response to this task can be based on any of these properties (or others not mentioned), and it may not be possible to tell in advance which property will lead to the response. Furthermore, it is likely that this discrimination is affected by other aspects of the task. For example, if subjects are required to make one response to either $\mathrm{X}$ or $\mathrm{O}$ and a different response to either $Y$ or $D$, they cannot rely exclusively on a single property, such as straight versus curved lines. By contrast, when subjects are required to make one response to $\mathrm{X}$ or $\mathrm{Y}$ and a different response to $\mathrm{O}$ or $\mathrm{D}$, they can rely exclusively on the presence or absence of closure (see Wolfe \& Bennett, 1997, for a more general discussion of this issue).

Given this situation, we assume that subjects try to make the required discrimination as simple as possible and therefore rely on simple properties if possible, rather than attempt to identify the whole letter. We further assume that the general visual context may affect the subjects' choice of the discriminating property. To illustrate the possible implications of these two assumptions, let us examine Versions 1 and 2 of Experiment 5. The subjects in both versions were asked to discriminate between the letters $S$ and F. They could have used a number of visual properties to make this discrimination successfully. They might have used, for instance, the property of straight versus curved lines. In Version 1 , the target $\mathrm{S}$ appeared with the flanker $\mathrm{O}$ on $90 \%$ of its trials, as did the target $\mathrm{F}$ with the flanker 
$\mathrm{X}$, whereas this pairing was reversed in Version 2. Given that $O$ is curved and $X$ is straight, in Version 1 a decision to discriminate between the target letters on the basis of this property would, in effect, have created congruent trials (i.e., the target appears in both target and flanker positions) in $90 \%$ of the cases and incongruent trials in $10 \%$ of the cases. By contrast, in Version 2 this decision would have created congruent trials in only $10 \%$ of the cases and incongruent trials in $90 \%$ of the cases. Because the task is easier for congruent trials, we propose that the subjects tended to choose the strategy of focusing on straight versus curved lines in Version 1, as this increases the proportion of congruent trials. By contrast, they opted for a different strategy (e.g., identifying the whole letter) in Version 2, to avoid creating a large proportion of incongruent trials.

The preceding paragraph offered a specific example of the general explanation we propose. The general notion is that subjects will tend to choose a discrimination property that will (1) make the required discrimination relatively easy and (2) take the visual context into consideration. In the context of the correlational cuing paradigm, they will have a higher tendency to choose a property that increases the congruency between target and flankers. This congruency is not necessarily an all-or-none affair. In Experiment 5, the degree of congruency appeared to be higher in Version 3 than in Version 1. Congruency in the flanker task may be a monotonic function of degree of similarity (see B. A. Eriksen \& C. W. Eriksen, 1974).

This explanation can accommodate the findings of the present study, as well as those of Miller (1987). One factor in the CE is the on-line processes (e.g., the repetition priming shown in Experiment 3 ) that occur while training with the correlation. These processes explain the findings with color and orientation targets, as well as those with dissimilar target-flanker letter pairs. A second factor, present with complex visual entities such as letters, is the choice of the visual property that is used for the discrimination. When similar target-flanker pairs are correlated, subjects may choose a property that is present in both the target and similar flankers (e.g., a straight line for target $F$ when correlated with $\mathrm{T}$ ). This will lead to an increase in the $\mathrm{CE}$, because both on-line local processes (e.g., repetition priming) and flanker congruency contribute to the $\mathrm{CE}$.

This explanation also accounts nicely for the transfer to explicit association obtained by Miller (1987, Experiment 3) and in Experiment 6 of the present study. To the extent that subjects respond to a visual property shared by the target and flankers, the presentation of the flanker is functionally equivalent to the presentation of the target. Thus, with our explanation, there is no transfer from implicit association to explicit association. Instead, subjects are faster in the transfer block when the (functional) target remains with the same assigned response than when it is assigned the alternative response. From our perspective, then, there is no tension between this type of transfer and the dissociation described earlier between implicit and explicit response codes (Cohen et al., 1995).

Admittedly, our explanation is speculative. Although the data are compatible with this explanation, some of its assumptions are not directly supported by the data. Moreover, because the similarity metric of letters is largely unknown, we cannot determine with certainty the candidate visual properties that may be chosen by subjects to discriminate among letters. Consequently, our choice of similar and dissimilar letters relied on implicit assumptions concerning the similarity metric for which no data are available. Furthermore, the open-ended nature of the claim that subjects can choose whatever property maximizes the efficiency of the task allows for post hoc rather than principled accounts. Nevertheless, we believe that this explanation at a general level does capture the essence of the findings.

Finally, preexperimental similarity between the target and the flankers is important for color targets as well. The subjects had an a priori tendency to respond faster to two of the target-flanker pairs. Second, although we did not find a statistically significant transfer with the easy pairs in Experiment 1, there appeared to be a tendency in this direction. It is possible, therefore, that similarity plays a role (albeit a minor one) for color targets, as it does for letter targets. However, the data are not conclusive, and more research is needed to determine whether and to what extent similarity plays a role in transfer of $\mathrm{CE}$ with color targets.

\section{Implications for Other Studies}

The present study may have important implications for studies with the correlational cuing paradigm. First, it shows that CE may not be an instance of learning at all and, instead, may be due to a host of local on-line processes that occur during the correlation manipulation. Second, it clearly demonstrates that the nature of the stimuli used in the task and the perceptual similarity between the set of target stimuli and the set of flanker stimuli are crucial. Random sampling, as was used by Miller (1987), may mask these effects and thus lead to wrong conclusions. Several examples may illustrate these points.

Carlson and Flowers (1996) used the correlational cuing paradigm to examine differences between intentional and unintentional types of learning, by manipulating instructions to the subjects concerning the existence of the correlation (informed vs. uninformed) and temporal presentation of target and flankers. No evidence of instruction manipulation was found when the target and flankers were presented simultaneously, as in a typical correlational cuing paradigm. In contrast, a difference was found when the flankers preceded the target or when contingencies between successive trials were manipulated. Carlson and Flowers concluded that intentional and unintentional types of learning have a different sensitivity to temporal events. However, they required subjects to discriminate between letters and digits and correlated the two categories with other characters. In such a design, it is impossible to estimate the nature of the target-flanker similarity. Moreover, our study suggests that the CE in the simultaneous presentation of target and flankers is not due to learning processes. Thus, the conclusions reached by Carlson and Flowers need to be qualified. 
Paquet and Craig (1997) examined the effect of semantic categories on correlational cuing by requiring subjects to discriminate among letters flanked by either nontarget letters or digits. They showed that manipulation of target cuing affected letter flankers differently than it affected digit flankers (see, also, Paquet \& Lortie, 1990). However, both letters and digits were selected randomly for each subject. In addition, the similarity of letter targets with letter and digit flankers was not matched. Thus, it is impossible to know whether the results obtained by Paquet and Craig are due to the influence of semantic categories on selection, as they suggest, or to differences in targetflanker similarity.

The correlational cuing paradigm has also been used to infer processes of selective attention (see, e.g., Miller, 1987, Paquet \& Lortie, 1990; Schmidt \& Dark, 1998). As was mentioned before, the correlational cuing paradigm is often advocated as an improvement over the flanker paradigm, because the distractors in the correlational cuing paradigm are not directly related to the task. Our findings indicate, however, that much of the $\mathrm{CE}$ is due to on-line processes. Although more research is required to determine the nature of these processes, it is possible that they are caused by relatively early perceptual processes and thus may be compatible with both early and late selection theories of attention. The additional CE observed for similar target-flanker pairs (Versions 1 and 3 of Experiment 5, as well as Experiment 6) may be caused by higher level processes. However, as suggested by our explanation, this component may be due to the flankers being functionally similar to the targets. If so, this effect is, in fact, identical to that observed in typical flanker tasks and can be triggered with equal efficiency by both paradigms.

An important qualification for our study, however, may also be in order. All the experiments in our study used a simple stimulus-to-response mapping (two stimuli to two responses). The subjects in this simple task situation may not seek to extract additional redundancies, and that may be the reason for the lack of learning. It is possible that, in more complex tasks, subjects will more actively seek redundancies in the stimulus displays, and learning may be obtained in such situations (see, e.g., Carlson \& Flowers, 1996). More research is required to evaluate this possibility. Our study clearly illustrates, however, that control of similarity between target and flankers and direct evaluation of learning (e.g., by a transfer block with no correlation) are necessary for a demonstration of true learning.

Finally, the conclusions from our study apply specifically to the correlational cuing paradigm in which subjects know in advance (and presumably, focus their attention on) the location of the target. We do not wish to claim that there is no implicit learning of contingencies between target and distractors in other situations. Indeed, recent research by Flowers and Smith (1998) convincingly demonstrated that subjects may be able to implicitly learn correlations between targets and distractors during visual search.

\section{REFERENCES}

Bertelson, P. (1963). S-R relationships and reaction time to new versus repeated signals in a serial task. Journal of Experimental Psychology, 65, 478-484.

BIEDERMAN, I. (1987). Recognition-by-components: A theory of human image understanding. Psychological Review, 94, 115-147.

Carlson, K. A., \& Flowers. J. H. (1996). Intentional versus unintentional use of contingencies between perceptual events. Perception \& Psychophysics, 58, 460-470.

Cohen, A., Ivry, R. B., Rafal, R. D., \& Kohn, C. (1995). Activating response codes by stimuli in the neglected visual field. Neuropsychology, 2, 165-173.

Cohen, A., \& Shoup, R. E. (1993). Orientation asymmetry in the flanker task. Perception \& Psychophysics, 53, 693-703.

CoHen, A., \& Shoup, R. [E.] (1997). Perceptual dimensional constraints on response selection processes. Cognitive Psychology, 32, 128-181.

Duncan, J., \& Humphreys, G. W. (1989). Visual search and stimulus similarity. Psychological Review, 96, 433-458.

ERIKSEN, B. A., \& ErIKSEN, C. W. (1974). Effects of noise letters upon the identification of a target letter in a nonsearch task. Perception \& Psychophysics, 16, 143-149.

ERIKSEN, C. W., \& ERIKSEN, B. A. (1979). Target redundancy in visual search: Do repetitions of the target within the display impair processing? Perception \& Psychophysics, 26, 195-205.

Flowers, J. H., \& SмIтH, K. L. (1998). What is learned about nontarget items in simple visual search. Perception \& Psychophysics, 60, 696-704.

Gibson, E. J. (1969). Principles of perceptual learning and development. New York: Appleton-Century-Crofts.

Kahneman, D., \& Treisman, A. M. (1984). Changing views of attention and automaticity. In R. Parasuraman \& D. R. Davies (Eds.), Varieties of attention (pp. 29-61). Orlando, FL: Academic Press.

KoRNBLUM, S. (1969). Sequential determinants of information processing in serial and discrete choice reaction time. Psychological Review, 76, 113-131.

LaviE, N. (1995). Perceptual load as a necessary condition for selective attention. Journal of Experimental Psychology: Human Perception \& Performance, 21, 451-468.

Maljkovic, V., \& Nakayama, K. (1994). Priming of pop-out: I. Role of features. Memory \& Cognition, 22, 657-672.

Miller, J. (1987). Priming is not necessary for selective-attention failures: Semantic effects of unattended, unprimed letters. Perception $\&$ Psychophysics, 41, 419-434.

MulLer, J. (1991). The flanker compatibility effect as a function of visual angle, attentional focus, visual transients, and perceptual load: A search for boundary conditions. Perception \& Psychophysics, 49, 270-288.

MORDKOFF, J. T. (1996). Selective attention and internal constraints: There is more to the flanker effect than biased contingencies. In A. Kramer, M. G. H. Coles, \& G. Logan (Eds.), Converging operations in the study of visual selective attention (pp. 483-502). Washington, DC: American Psychological Association.

MORDKOFF, J. T., \& YANTIS, S. (1991). An interactive race model of divided attention. Journal of Experimental Psychology: Human Perception \& Performance. 17, 520-538.

PAQuet, L., \& CraIG, G. L. (1997). Evidence for selective target processing with a low perceptual load flankers task. Memory \& Cognition, 25, 182-189.

Paquet, L., \& Lortie, C. (1990). Evidence for early selection: Precuing target location reduces interference from same-category distractors. Perception \& Psychophysics, 48, 382-388.

Prinzmetal, W., Presti, D. E., \& Posner, M. I. (1986). Does attention affect visual feature integration? Journal of Experimental Psychology: Human Perception \& Performance, 12, 361-369.

RoBertson, L. C. (in press). Attentional persistence for features of hierarchical patterns. Journal of Experimental Psychology: General.

SCHMIDT, P. A., \& DARK, V. J. (1998). Attentional processing of "unattended" flankers: Evidence for a failure of selective attention. Perception \& Psychophysics, 60. 227-238. 
TownSEND, J. T. (1971). Theoretical analysis of an alphabetic confusion matrix. Perception \& Psychophysics, 9, 40-50.

Treisman, A. M., \& Gelade, G. (1980). A feature integration theory of attention. Cognitive Psychology, 12, 97-136.

VAN DER HeIJDEN, A. H. C. (1981). Short-term visual information forgetting. London: Routledge \& Kegan Paul.

WolfE, J. M., \& BenNETT, S. C. (1997). Preattentive object files: Shapeless bundles of basic features. Vision Research, 37, 25-43.

YANTIS, Y., \& Johnston, J. C. (1990). On the locus of visual selection: Evidence from focused attention tasks. Journal of Experimental Psychology: Human Perception \& Performance, 16, 135-149.

\section{NOTES}

1. The results are qualitatively the same when the two easy pairs (blue-green, yellow-red) and the two difficult pairs (blue-red, yellow- green) are examined separately. The mean RTs for the easy pairs were $490,506,516$, and $507 \mathrm{sec}$ for the STSF, STDF, DTSF, and DTDF conditions, respectively. The mean RTs for the difficult pairs were 488,502 , 512 , and $513 \mathrm{msec}$ for the STSF, STDF, DTSF, and DTDF conditions, respectively. Because of the small number of trials, we did not analyze these conditions statistically.

2. We changed the distance between the target and the flankers to make our design more similar to that of Miller (1987). To make sure that the change in distance is not critical, we also ran the first two versions of Experiment 5 with the same center-to-center distance as that in the previous experiments. Although the magnitude of the $C E$ was smaller than that in Experiment 5, the pattern of the results was essentially identical to that obtained with the smaller distance.

(Manuscript received July 14, 1997; revision accepted for publication January 28, 1998.) 